\title{
Does Surgery for Adult Spinal Deformity Affect the Cognitive Abilities in Patients over 50 Years of Age?
}

\author{
Vugar NABIYEV ${ }^{1}$, Selim AYHAN ${ }^{1}$, Selcen YUKSEL ${ }^{2}$, Asli NIYAZI ${ }^{3}$, Tiro MMOPELWA ${ }^{1}$, Montse DOMINGO-SABAT ${ }^{4}$, \\ Alba VILA-CASADEMUNT ${ }^{4}$, Ferran PELLISE ${ }^{4}$, Ahmet ALANAY ${ }^{5}$, Francisco J. Sanchez PEREZ-GRUESO ${ }^{6}$, \\ Frank KLEINSTUCK ${ }^{7}$, Ibrahim OBEID ${ }^{8}$, Emre ACAROGLU1', European Spine Study Group (ESSG) ${ }^{4}$ \\ ${ }^{1}$ Acibadem ARTES Spine Center, Spine and Spinal Cord Surgery, Ankara, Turkey \\ ${ }^{2}$ Yildirim Beyazit University, Department of Biostatistics, Ankara, Turkey \\ ${ }^{3}$ Department of Psychology, Middle East Technical University, Northern Cyprus Campus, Guzelyurt, Mersin 10, Turkey \\ ${ }^{4}$ Hospital Universitari Vall d'Hebron, Spinal Unit, Barcelona, Spain \\ ${ }^{5}$ Acibadem Maslak Hospital, Comprehensive Spine Center, Istanbul, Turkey \\ ${ }^{6}$ Hospital Universitari La Paz, Spine Unit, Madrid, Spain \\ ${ }^{7}$ Schultess Clinic, Musculoskletal Center, Zurich, Switzerland \\ ${ }^{8}$ Bordeaux University Hospital, Department of Spine Surgery, Bordeaux, France
}

\section{ABSTRACT}

AIM: To analyze the cognitive abilities of older patients undergoing spinal deformity surgery so as to understand whether adult spinal deformity (ASD) surgery is associated with postoperative cognitive malfunction.

MATERIAL and METHODS: A prospective longitudinal study was performed on surgical patients older than 50 years, enrolled in a prospective multicenter database. Mini-mental state examination (MMSE) was performed to assess cognitive function in addition to the health-related quality of life (HRQOL) tests; (SF-36, ODI and SRS-22) at the pre-operative, post-operative 6 weeks and 6 months points. Demographics, preoperative health status, co-morbidities and surgical characteristics were also analyzed. Descriptive statistics and repeated measures of variance analysis were performed.

RESULTS: A total of 90 patients with a mean age of $67.4 \pm 8.2$ years were enrolled in the study; all had MMSE evaluations at 6 weeks follow-up and 58 patients had both 6 weeks and 6 months follow-up. Average (SD) surgical time, estimated blood loss, number of levels fused and hospital stay were 240.1 (111.9) minutes, 1621.2 (1058.7) ml, 11.2 (4.4) levels and 14.2 (11.45) days, respectively. On analysis, a slight increase in mean MMSE score $(p>0.05)$ between time points was found despite decreases of $>2$ points $(3$ or 4$)$ in 6 patients $(6.7 \%)$ at both time points.

CONCLUSION: Although ASD surgery in older patients is recognized as challenging, this study suggests that it is not necessarily associated with a significant deterioration in the cognitive abilities of patients undergoing surgery. This may be due to the relatively minor influence of ASD itself on the cognitive abilities of the patients involved as well as to the relatively stable hemodynamic conditions obtainable during modern ASD surgery.

KEYWORDS: Adult spinal deformity, Cognitive abilities, Non-cardiac, MMSE, Surgery 


\section{INTRODUCTION}

$\mathrm{S}$ pinal deformity surgery is among common major surgeries performed on older adults (17). The goal of treatment is to achieve deformity correction, pain relief and neurological improvement while minimizing the surgical complications which tend to be fairly common as well (15).

Elderly patients undergoing major surgery may experience cognitive deterioration due to a decreased plasticity in their brain tissue. This so called "postoperative cognitive dysfunction (POCD) syndrome" is characterized by nonspecific dysfunction in memory, concentration and analysis skills (10). Older age, lower level of education, previous cerebrovascular accident without residual impairment and postoperative cognitive dysfunction at discharge have been suggested as the independent factors for POCD at 3 months $(1,11)$. Postoperative delirium and increased use of opioid analgesics were also significantly correlated with POCD at hospital discharge, but not at the $3^{\text {rd }}$ month (2). In addition, patients who had POCD have also been shown to be at increased risk of death in the first year after surgery (4). Major surgery, history of alcohol abuse in elderly, postoperative changes in thyroid hormones, Alzheimer's disease, cerebral trauma and preoperative cognitive decline are the other risk factors for the development of $\operatorname{POCD}(5,6,8,13)$. In another study, it has been demonstrated that the risk of dementia and mild cognitive impairment in hip surgery patients who are 70 years and older was almost doubled if they had postoperative delirium as compared to those who had not (14). On the other hand, interestingly, it has also been fairly well documented that early and late POCD is related to the overall "success" of surgery in elderly patients (9).

The aim of this study was to analyze the cognitive abilities of older patients undergoing surgery for spinal deformity at the specific time points of 6 weeks (early post-op) and 6 months (late post-op), so as to understand whether such major surgery might be associated with long term and potentially permanent POCD.

\section{MATERIAL and METHODS}

From March 2010 to June 2014, a prospective longitudinal study was conducted on an adult spinal deformity (ASD) population from all sites of the study group. The criteria to be included in this multicentric study database were defined as the patient having any of (or any combination of) the following: thoracic kyphosis exceeding $60^{\circ}$, coronal deformity exceeding $20^{\circ}$, pelvic tilt exceeding $25^{\circ}$ and sagittal vertical axis exceeding $50 \mathrm{~mm}$.

A total of 90 patients who were 50 years of age or older, with grossly normal general intellect and free of dementia, depression or any history of cerebrovascular accident were included in the study. All the patients consented to be enrolled in the database as well as this separate study. The database including this individual study had Institutional Review Board (IRB) approval from all participating centres.
Mini mental state scores (MMSE), individually validated for all languages used previously, were used to assess cognitive functions in addition to the health related quality of life (HRQOL) tests (SF-36, ODI and SRS-22) at preoperative, and 6 weeks and 6 months post-operative time points. Demographic data, preoperative health status, co-morbidities, and surgical characteristics were also analyzed and documented.

The diagnosis of cognitive impairment was made on the basis of the MMSE scores, which is a brief structured test with widely accepted validity as a measure of cognitive functioning (12). The MMSE includes 11 items that evaluate 5 different domains of cognitive functioning; orientation (in time and space, 10 points), registration (of 3 words, 3 points), attention and calculation (spelling a word backwards, 5 points), recall (remembering the 3 words, 3 points), and language (to name a pencil and a watch, to repeat a sentence, to understand and effect an oral 3-step instruction and a written instruction, to write a sentence and finally to draw a geometric figure; in all, 9 points) (3). In this test, the maximum available score is 30 points. Scores from 27 to 30 are considered as normal, from 18 to 26 as mild, from 10 to 17 as moderate, and below 10 as severe cognitive dysfunction (7). This tool has been shown to have good test-retest reliability (16).

However, a diagnosis of POCD cannot simply be based on any absolute score value on this test, but rather interpreted as a significant decrease in MMSE score postoperatively compared to the preoperative values, which was arbitrarily accepted as 3 points or more in this study.

\section{Statistical Analysis}

The sociodemographic characteristics of the patients as well as their hospital course data were descriptively analyzed by mean, percentages, minimal and maximal values. Differences between the baseline characteristics of patients with and without cognitive deficits were tested using Student's t-test for repeated measures and the likelihood ratio statistic test for proportion measures. These differences were examined using assessments at both 6 weeks and 6 months after surgery. The results for the MMSE were analyzed to assess whether cognitive function and health-related quality of life outcome measures had changed between the preoperative test and the six weeks and six months postoperative period. A $p$ value $<0.05$ was considered as significant. The calculated power of this study at this $\beta$ type error level was $99 \%$.

\section{RESULTS}

Summary of demographic and surgical data for these samples are provided in Table I. As can be seen from the surgical data, surgeries performed on this group of patients have been very diverse, ranging from 1 to 20 levels (mean 4.4) of segmental instrumentation with an average blood loss of over $1600 \mathrm{ml}$ (range 200 to 4800).

As for the cognitive analyses by MMSE, there was no discernible change in performance in the majority of patients at the $6^{\text {th }}$ week compared to the baseline tests. As can be seen in Table II, MMSE scores even show a slight increase in mean 
score $(p>0.05)$ between these time points (Figure 1). A total of 58 patients had their functions assessed at 6 months as well. Again at this time point, the MMSE test did not show evidence of clinically significant deterioration in cognitive function in long-term post-operative follow-up (Figure 1), however there was a decrease of $>2$ points ( 3 or 4 ; arbitrarily defined as the threshold of deterioration) in 6 patients $(6.7 \%)$ at both time points (Table III).

When the patients were divided into two groups as elderly (>65 years) or not, no statistically significant difference was seen in inter- and intragroup comparisons at 6 months followup ( $p>0.05$ ) (Table IV) although the preoperative scores were different between the two groups $(p=0.006)$. Of note, being elderly (>65 years) was not found to cause a significant risk of a decline in MMSE scores of more than two points either (Table V).

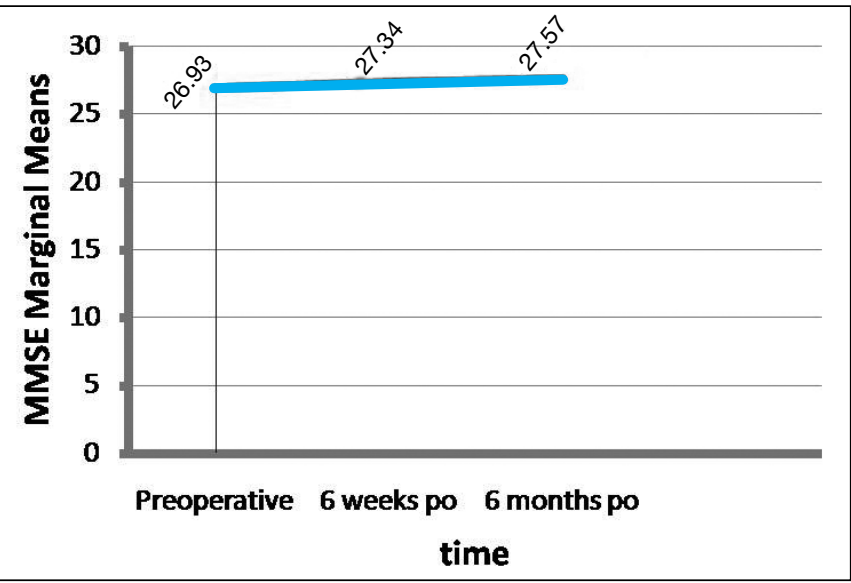

Figure 1: Mean MMSE scores of patients who underwent adult spinal deformity surgery; preoperative and at 6 weeks and 6 months time points postoperative.

Table I: Demographical and Surgical Characteristics of the Patients $(n=90)$

\begin{tabular}{|c|c|c|c|}
\hline Value & n (\%) & Mean (min-max) & Std Dev \\
\hline \multicolumn{4}{|l|}{ Demographical Characteristic } \\
\hline Gender (Female/Male) & $71(78.9) / 19(21.1)$ & & \\
\hline Age (years) & & 67.4 & 8.2 \\
\hline \multicolumn{4}{|l|}{ Etiology } \\
\hline Degenerative & $36(40)$ & & \\
\hline Idiopathic & $34(37.8)$ & & \\
\hline Others* & $20(22.2)$ & & \\
\hline \multicolumn{4}{|l|}{ Surgical Characteristic } \\
\hline Surgical Time (minutes) & & $240.1(75-680)$ & 111.9 \\
\hline Estimated blood loss (ml) & & $1621.2(200-4800)$ & 1058.7 \\
\hline Osteotomy (Yes/no) & 55 (61.1) / 35 (38.9) & & \\
\hline Number of levels fused & & $11.2(1-20)$ & 4.4 \\
\hline Length of hospitalization (day) & & $14.2(3-84)$ & 11.45 \\
\hline
\end{tabular}

*Others: failed-back, post-traumatic, Scheuermann, neuromuscular, congenital, post-infection.

Table II: Mean MMSE Scores of Patients

\begin{tabular}{llcll}
\hline $\mathbf{A}$ & Mean & Std. Deviation & $\mathbf{n}$ & $\mathbf{p}$ \\
\hline Preoperative & 26.88 & 2.691 & 90 & 0.158 \\
\hline $6^{\text {th }}$ weeks po & 27.17 & 2.474 & 90 & $\mathbf{n}$ \\
\hline $\mathbf{B}$ & Mean & Std. Deviation & 58 & \\
\hline Preoperative & 26.93 & 2.815 & 58 & 0.998 \\
\hline $6^{\text {th }}$ weeks po & 27.34 & 2.579 & 58 & 0.06 \\
\hline $6^{\text {th }}$ months po & 27.57 & 2.528 & 5.92 & \\
\hline
\end{tabular}

MMSE: Mini mental state examination, po: Postoperative, A: Patients with 6 weeks follow-up; B: Patients with 6 months follow-up. 


\section{DISCUSSION}

This study has investigated the potential for cognitive impairment and POCD following major surgery in a population of ASD aged over fifty years. Preoperative, $6^{\text {th }}$ week and $6^{\text {th }}$ month MMSE scores were compared, and no significant difference could be found. Of note, 6 patients had deterioration more than the arbitrarily assigned significance level of 3 points or more, but none had decreased below the level of mild impairment. These findings suggest that contrary to the intuitive perception of cognitive impairment in relatively older patients undergoing major spinal deformity surgery, very few patients are affected at all and none develop significant impairment.

Apart from the studies cited above, the first wide-ranging prospective study was conducted by a multicenter research

Table III: Demographical and Surgical Characteristics of 6 Patients Whose MMSE Scores Decreased $>2$ Points at 6 Months

\begin{tabular}{ccccccccc}
\hline $\begin{array}{c}\text { Patient } \\
\#\end{array}$ & $\begin{array}{c}\text { Age } \\
\text { (y) }\end{array}$ & Gender & Etiology & $\begin{array}{c}\text { Surgical time } \\
\text { (minutes) }\end{array}$ & $\begin{array}{c}\text { Estimated blood } \\
\text { loss (milliliters) }\end{array}$ & $\begin{array}{c}\text { Osteotomy } \\
\text { (yes/no) }\end{array}$ & $\begin{array}{c}\text { Number of } \\
\text { levels fused }\end{array}$ & $\begin{array}{c}\text { Length of } \\
\text { hospitalization (days) }\end{array}$ \\
\hline 1 & 55 & M & FB & 180 & 2300 & Yes & 9 & 11 \\
\hline 2 & 59 & F & C & 135 & 1159 & Yes & 13 & 9 \\
\hline 3 & 66 & F & i & 225 & - & No & 12 & 20 \\
\hline 4 & 69 & M & D & 180 & - & No & 16 & 9 \\
\hline 5 & 75 & F & D & 145 & 800 & Yes & 12 & 3 \\
\hline 6 & 75 & F & D & 240 & 1200 & Yes & 15 & 2 \\
\hline
\end{tabular}

C: Congenital, D: Degenerative, F: Female, FB: Failed-back, I: Idiopathic, M: Male, y: Years.

Table IV: Mean MMSE Scores of Patients being 65 Years-Old or Younger and Older

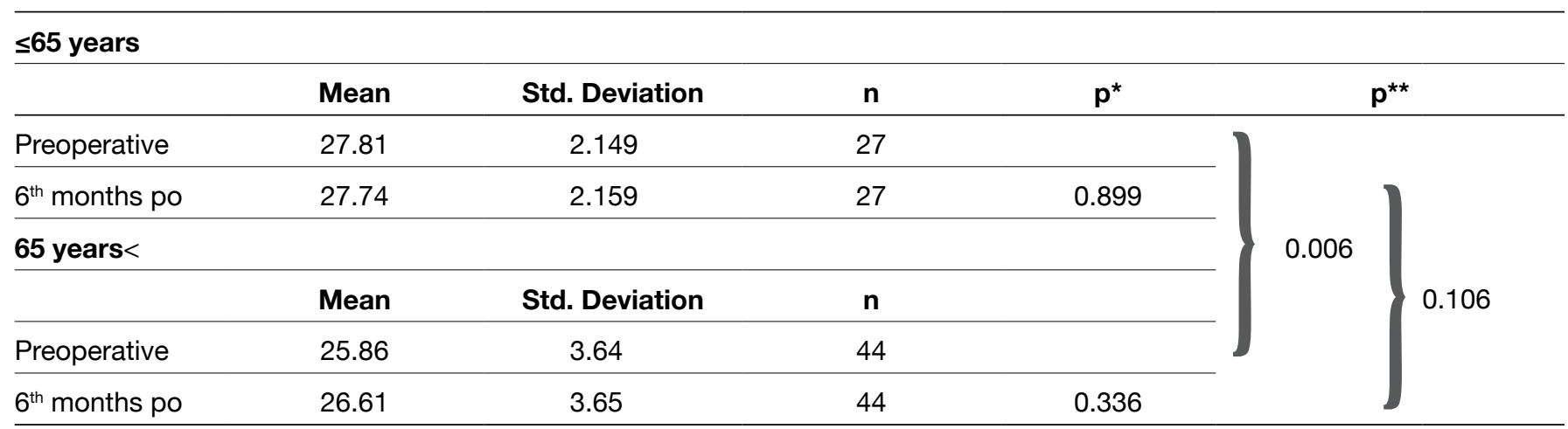

MMSE: Mini mental state examination, po: Postoperative.

*Intra-group comparisons (Student's t-test).

**Inter-group comparisons (Student's t-test).

Table V: The Relationship Between the Decline of MMSE Scores and the Age Being 65 Years or Younger and Older

\begin{tabular}{|c|c|c|c|c|}
\hline \multicolumn{5}{|l|}{ A } \\
\hline $0-2$ & 15 & & 13 & \\
\hline$\geq 3$ & 3 & & 5 & 0.42 \\
\hline \multicolumn{5}{|l|}{ B } \\
\hline $0-2$ & 7 & & 6 & \\
\hline$\geq 3$ & 1 & & 5 & 0.13 \\
\hline
\end{tabular}

MMSE: Mini mental state examination, po: Postoperative, A: Patients with 6 weeks follow-up, B: Patients with 6 months follow-up. ${ }^{*}$ Chi-square test. 
group on patients undergoing non-cardiac surgery (9). The psychometric test results for the first and third postoperative months were analysed for patients above 60 years of age undergoing major abdominal and orthopaedic surgery in this study and the authors reported POCD in nearly $25 \%$ of the patients at the time of discharge and $10 \%$ at the postoperative third month. In addition, the POCD rate in a recently published study has been reported in patients undergoing coronary arteriography (under sedation) or total hip replacement or cardiac by-pass surgery (under general anesthesia) to be as high as $17 \%$ (4). This study also suggested that the development of POCD was related to the type of surgery and type of anesthesia (elevated in general anesthesia and by-pass surgery).

This brief summary of the literature leads to a question on the reasons why our findings are not consistent with these. One hypothesis may be the fact that the surgeries performed on this series of patients may not be consistently as aggressive as other surgeries in those reports, especially cardiac by-pass surgery. This may be correct when analysed on average, but as demonstrated by the wide range of the surgical spectrum included in this study, it is still surprising that none of our patients had deteriorated below the even mild level of cognitive dysfunction and again, none had deteriorated more than 4 points on the MMSE. We therefore tend to think that spinal surgery, although potentially being very aggressive at times, may not be very conducive to the development of POCD, probably because of the relatively stable haemodynamics and tissue oxygenation throughout surgery (contrary to coronary by-pass surgery or even total hip replacement surgery). This remains a speculation that may warrant further investigation.

Another possible reason may the relatively minor influence of ASD by itself on the cognitive abilities of the involved population. In this respect, the most important factor may be the co-morbidities associated with ASD in patients undergoing spinal surgery. Although this has not been specifically addressed in this present study, our perception based on our (almost) uniformly favorable results in regard to the retainment of cognitive abilities (regardless of the potential co-morbidities) causes us to think that such an analysis might not have yielded further support to this hypothesis as well. Namely, since there were no real deteriorations demonstrated in this series of patients with a wide array of co-morbidities, there is probably no reason to analyse the specific risks attributable to specific co-morbidities. In this regard, the present authors tend to think that the apparent differences between this and previous studies on seemingly similar surgeries arise from the misperception of similarity. ASD surgery, albeit complex, does not genuinely seem to affect cognitive function.

At this point, it has to be underlined that this study has not demonstrated that there is no detrimental effect of surgery in any of the patients. Six (6.7\%) patients did demonstrate decreases over the threshold we had defined ( $>2$ points) but then again pretty much stayed just above this threshold (i.e. 3 or 4 points). This finding is important in the sense that it underlines the aggressiveness of the surgery performed, and than that there is no major deterioration encountered.
This study aimed to report the relative incidence of POCD in a specific patient population undergoing a specific type of surgery. We did not attempt to understand the mechanisms underlying cognitive decline as well as delirium by way of additional biomechanical and/or functioning imaging studies. Albeit humble, we still believe this information is important for spine surgeons performing deformity surgery in adults for both academic and medico-legal reasons.

\section{CONCLUSION}

Although ASD surgery in older patients is recognized as challenging and aggressive, our study suggests that it is not necessarily associated with a significant deterioration in the cognitive abilities of the patients. These results are different from those previously reported by other investigators regarding other major surgical interventions. This may be due to the relatively minor influence of ASD itself on the cognitive abilities of the patients involved as well as to the relatively stable hemodynamic conditions obtainable during modern ASD surgery.

\section{ACKNOWLEDGEMENT}

This project has been funded by a research grant from Depuy Synthes directed to the European Spine Study Group (ESSG).

\section{REFERENCES}

1. Bhagat S, Vozar V, Lutchman L, Crawford RJ, Rai AS: Morbidity and mortality in adult spinal deformity surgery: Norwich Spinal Unit experience. Eur Spine J 22 Suppl 1:S42-46, 2013

2. Bickel $H$, Gradinger R, Kochs E, Forstl H: High risk of cognitive and functional decline after postoperative delirium. A threeyear prospective study. Dement Geriatr Cogn Disord 26:2631, 2008

3. Duppils GS, Wikblad K: Cognitive function and health-related quality of life after delirium in connection with hip surgery. A six-month follow-up. Orthop Nurs 23:195-203, 2004

4. Evered L, Scott DA, Silbert B, Maruff P: Postoperative cognitive dysfunction is independent of type of surgery and anesthetic. Anesth Analg 112:1179-1185, 2011

5. Goto T, Baba T, Honma K, Shibata Y, Arai Y, Uozumi H, Okuda $\mathrm{T}$ : Magnetic resonance imaging findings and postoperative neurologic dysfunction in elderly patients undergoing coronary artery bypass grafting. Ann Thorac Surg 72:137-142, 2001

6. Hudetz JA, lqbal Z, Gandhi SD, Patterson KM, Hyde TF, Reddy DM, Hudetz AG, Warltier DC: Postoperative cognitive dysfunction in older patients with a history of alcohol abuse. Anesthesiology 106:423-430, 2007

7. Koponen HJ, Riekkinen PJ: A prospective study of delirium in elderly patients admitted to a psychiatric hospital. Psychol Med 23:103-109, 1993

8. Mafrica F, Fodale V: Thyroid function, Alzheimer's disease and postoperative cognitive dysfunction: a tale of dangerous liaisons? J Alzheimers Dis 14:95-105, 2008 
9. Moller JT, Cluitmans P, Rasmussen LS, Houx P, Rasmussen H, Canet J, Rabbitt P, Jolles J, Larsen K, Hanning CD, Langeron O, Johnson T, Lauven PM, Kristensen PA, Biedler A, van Beem $\mathrm{H}$, Fraidakis O, Silverstein JH, Beneken JE, Gravenstein JS: Long-term postoperative cognitive dysfunction in the elderly ISPOCD1 study. ISPOCD investigators. International Study of Post-Operative Cognitive Dysfunction. Lancet 351:857-861, 1998

10. Monk TG, Price CC: Postoperative cognitive disorders. Curr Opin Crit Care 17:376-381, 2011

11. Monk TG, Weldon BC, Garvan CW, Dede DE, van der Aa MT, Heilman KM, Gravenstein JS: Predictors of cognitive dysfunction after major noncardiac surgery. Anesthesiology 108:18-30, 2008

12. Patten SB, Fick GH: Clinical interpretation of the mini-mental state. Gen Hosp Psychiatry 15:254-259, 1993
13. Rasmussen LS, Siersma VD: Postoperative cognitive dysfunction: True deterioration versus random variation. Acta Anaesthesiol Scand 48:1137-1143, 2004

14. Scott JE, Mathias JL, Kneebone AC: Postoperative cognitive dysfunction after total joint arthroplasty in the elderly: A metaanalysis. J Arthroplasty 29:261-267.e261, 2014

15. Takahashi S, Delecrin J, Passuti N: Surgical treatment of idiopathic scoliosis in adults: An age-related analysis of outcome. Spine (Phila Pa 1976) 27:1742-1748, 2002

16. Tombaugh TN, Mclntyre $\mathrm{NJ}$ : The mini-mental state examination: A comprehensive review. J Am Geriatr Soc 40:922-935, 1992

17. Weiss HR, Goodall D: Rate of complications in scoliosis surgery - a systematic review of the Pub Med literature. Scoliosis 3:9, 2008 